\title{
COVID-19 mRNA vaccine induced rhabdomyolysis and fasciitis
}

\author{
Simon Faissner ${ }^{1} \mathbb{C} \cdot$ Daniel Richter $^{1} \cdot$ Ulas Ceylan $^{1} \cdot$ Christiane Schneider-Gold $^{1} \cdot$ Ralf Gold $^{1}$
}

Received: 9 August 2021 / Revised: 18 August 2021 / Accepted: 18 August 2021 / Published online: 25 August 2021

(c) The Author(s) 2021

Dear Sirs,

Since the end of 2019, the coronavirus disease 2019 (COVID-19) pandemic induced by an infection with severe acute respiratory coronavirus 2 (SARS-CoV-2) has led to millions of deaths worldwide. The unprecedented fast development of a vaccination against SARS-CoV-2 led to the approval of several vaccinations by the authorities since December 2020. Rare severe side effects are sometimes not observed during the pivotal trials but get noticed during daily clinical practice such as thrombotic thrombocytopenia after ChAdOx1 nCov-19 vaccination [1]. Thus, the description of rare side effects is urgently needed to optimize the worldwide COVID-19 vaccination campaign. Here, we report for the first time a case of myopathy with severe rhabdomyolysis and fasciitis following mRNA vaccination against SARS-CoV-2. The patient was treated in the University Hospital of Ruhr-University Bochum and gave written informed consent for the publication of her case.

A 28-year-old healthy female received the first dosage of mRNA vaccination against SARS-CoV-2 (Moderna). Five days later, she complained about muscle pain of her thigh muscles, radiating to the lower legs, accompanied by an asymmetrical weakness of the lower limbs. Seven days following vaccination, a blood test revealed marked elevation of creatine kinase and transaminases. At first presentation, she had a mild predominantly left-sided weakness of hip flexor and knee extension (MRC 4-/5 vs. MRC 4/5) with marked subcutaneous leg edema (Fig. 1a), the creatine kinase was 17,959 U/l (normal range 26-140 U/l). There had been no preceding muscular symptoms such as exercise intolerance or anesthetic reactions that might have suggested a preceding predisposition to develop rhabdomyolysis. There was also no relevant family history. Immediate workup

Simon Faissner

simon.faissner@rub.de

1 Department of Neurology, Ruhr-University Bochum, St. Josef-Hospital, Gudrunstr. 56, 44791 Bochum, Germany excluded infection with hepatitis viruses, EBV or CMV. An echocardiography and a thoracic computed tomography were unremarkable. The patient was immediately treated with high volume normal saline infusion and urine alkalization. Renal function gradually worsened with a creatinine of $1.02 \mathrm{mg} / \mathrm{dl}$ ( $75 \mathrm{ml} / \mathrm{min}$ glomerular filtration rate according to Cockcroft-Gault) on day 5 of in-hospital treatment. Moreover, hypocalcemia of $1.97 \mathrm{mmol} / \mathrm{l}$ and moderate hypophosphatemia of $0.38 \mathrm{mmol} / \mathrm{l}$ were detected at first presentation, which gradually resolved within 4 days; parathormone was normal. Antibodies associated with myositis or myopathy were all negative (Mi-2a/b, TIF1g, MDA5, NFP2, SAE1, Ku, PM-Scl100, PM-Scl75, Jo-1, SRP, PL-7, PL-12, EJ, OJ, Ro-52, cN-1A). An MRI of the thigh muscles, performed on the following day, showed left-dominant edematous signal alterations with contrast enhancement of the quadriceps muscles sparing the M. rectus femoris, and diffuse subcutaneous fluid retention with contrast enhancement, suggestive of fasciitis (Fig. 1b, c). Electromyography of the left rectus muscle showed positive sharp waves and fibrillations with small and partly polyphasic motor unit potentials, compatible with an acute myopathy or myositis. The patient was treated with an i.v. cycle of $250 \mathrm{mg}$ methylprednisolone over 2 days, leading to complete remission of paresis, leg pain and remission of leg edema within days, followed by oral tapering (60 mg methylprednisolone orally, tapered over 6 days). Four weeks after onset, creatine kinase was normal, the weakness and leg edema were gone, and she could do jogging again.

COVID-19 mRNA vaccine-associated side effects include pain, redness and pain at the injection site, fatigue, headache, myalgia or arthralgia [2,3]. One case described local COVID-19 vaccine-related myopathy in the deltoid muscle with probable myositis [4]. Recently, another case report described severe rhabdomyolysis 1 day after BioNTech/ Pfizer COVID-19 vaccine administration with creatinine kinase levels of up to 22,000 U/1 [5]; however, without signs of myopathy or fasciitis as found in the patient presented here. COVID-19 can induce a broad range of neurological symptoms including encephalitis, encephalopathy, cranial 
Fig. 1 COVID-19 mRNA vaccine induced myopathy with rhabdomyolysis and fasciitis in a 28-year-old female. a Edematous swelling of both legs. b Transversal MRI of the thigh muscles in $\mathrm{T} 2$ and c $\mathrm{T} 1$ post-contrast shows left dominant signal alterations of the quadriceps muscles with contrast enhancement and subcutaneous edema suggestive of accompanying fasciitis
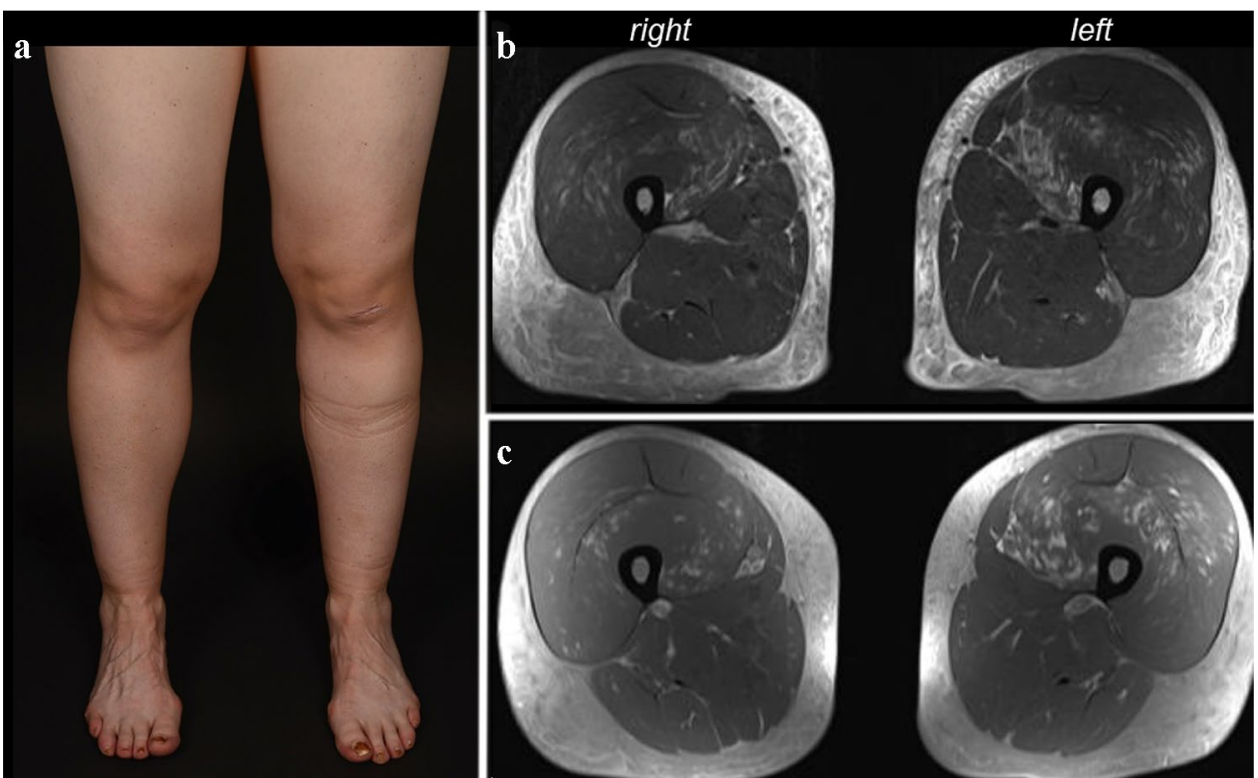

neuropathy, Guillain-Barré syndrome, and myositis/rhabdomyolysis. As of September 2020, Paliwal et al. found nine cases of myositis/rhabdomyolysis induced by natural COVID-19 disease [6]. One report documented a patient with myopericarditis and myositis with similar subcutaneous edema and signal alterations of the thigh muscles suggestive of myositis with creatine kinase elevations [7] as observed in our patient following vaccination, suggesting similar pathomechanisms.

In summary, we present a new and so far unknown complication of mRNA vaccination against SARS-CoV-2. Clinicians should be vigilant especially in patients developing myalgia with paresis following COVID-19 vaccination to detect rhabdomyolysis and start treatment without delay.

Acknowledgements We thank the patient for the permission to publish her case.

Author contributions Study design: SF and RG. Investigation: SF, DR, UC, CSG, and RG. Data collection: SF, DR, UC, and CSG. Data analysis: SF, DR, CSG, and RG. Data interpretation: SF, CSG, and RG. Methodology: SF, CSG, and RG. Figures: SF and DR. Writing-original draft: SF. Writing-review and editing: DR, UC, CSG, and RG.

Funding Open Access funding enabled and organized by Projekt DEAL.

Data availability Data are available from the corresponding author upon reasonable request.

\section{Declarations}

Conflicts of interest The authors declare no competing interests relevant to the content of this manuscript.
Ethical standard statement The patient provided written informed consent for the publication of the case report.

Open Access This article is licensed under a Creative Commons Attribution 4.0 International License, which permits use, sharing, adaptation, distribution and reproduction in any medium or format, as long as you give appropriate credit to the original author(s) and the source, provide a link to the Creative Commons licence, and indicate if changes were made. The images or other third party material in this article are included in the article's Creative Commons licence, unless indicated otherwise in a credit line to the material. If material is not included in the article's Creative Commons licence and your intended use is not permitted by statutory regulation or exceeds the permitted use, you will need to obtain permission directly from the copyright holder. To view a copy of this licence, visit http://creativecommons.org/licenses/by/4.0/.

\section{References}

1. Greinacher A et al (2021) Thrombotic thrombocytopenia after ChAdOx1 nCov-19 vaccination. N Engl J Med 384(22):2092-2101

2. Polack FP et al (2020) Safety and efficacy of the BNT162b2 mRNA COVID-19 vaccine. N Engl J Med 383(27):2603-2615

3. Baden LR et al (2021) Efficacy and safety of the mRNA-1273 SARS-CoV-2 vaccine. N Engl J Med 384(5):403-416

4. Theodorou DJ et al (2021) COVID-19 vaccine-related myositis. QJM. https://doi.org/10.1093/qjmed/hcab043

5. Nassar $\mathrm{M}$ et al (2021) COVID-19 vaccine induced rhabdomyolysis: case report with literature review. Diabetes Metab Syndr 15(4): 102170

6. Paliwal VK et al (2020) Neuromuscular presentations in patients with COVID-19. Neurol Sci 41(11):3039-3056

7. Shabbir A et al (2020) Myopericarditis and myositis in a patient with COVID-19: a case report. Eur Heart J Case Rep 4(6):1-6 